\title{
Magnetic Anisotropy of Epitaxially Grown Fe/Mn/Co Trilayers
}

\author{
Marcio Solino Pessoa ${ }^{1}$, Fernando Pelegrini ${ }^{1}$, Tales Costa de Freitas ${ }^{2}$, Edson Caetano Passamani ${ }^{2}$, \\ Sebastien Couet $^{3}$, Kristiaan Temst ${ }^{3}$, and André Vantomme ${ }^{3}$ \\ ${ }^{1}$ Instituto de Física, Universidade Federal de Goiás, Goiânia 74001-970, Brazil \\ ${ }^{2}$ Departamento de Física, Universidade Federal do Espírito Santo, Vitória 29075-910, Brazil \\ ${ }^{3}$ Instituut voor Kern-en Stralingsfysica, KU Leuven, B-3001 Leuven, Belgium
}

\begin{abstract}
This paper reports a study of cubic magnetic anisotropies of bcc-Fe and bcc-Co structures in Fe/Mn/Co trilayers grown by molecular beam epitaxy onto $\mathrm{MgO}(001)$ substrates. Parallel ferromagnetic resonance experiments, at microwave frequency of $33.9 \mathrm{GHz}$, reveal the presence of a large fourfold magnetic anisotropy in all studied films. Two uniform resonance modes, with fourfold magnetic anisotropy in both bcc-Fe and Co layers, are excited by the microwave field in samples with Mn thicknesses of 0.83 and $0.99 \mathrm{~nm}$. Films with thick Mn layer (1.16-2.20 nm) do not exhibit independent $\mathrm{Fe}$ and $\mathrm{Co}$ uniform modes, a result associated with an enhancement of the roughness at the $\mathrm{Mn} / \mathrm{Co}$ interface ( $\mathrm{Fe} / \mathrm{Mn}$ interface roughness was estimated by ${ }^{57} \mathrm{Fe}$ Mössbauer spectroscopy to be nearly constant for all films) at the $\mathrm{Fe} / \mathrm{Mn}$ and $\mathrm{Mn} / \mathrm{Co}$ interfaces. Magnetic anisotropy constants of bcc-Fe and bec-Co layers of sample with $0.99 \mathrm{~nm}$ thick $\mathrm{Mn}$ layer were determined, respectively, as $4.5 \times 10^{5}$ and $6.4 \times 10^{5} \mathrm{erg} / \mathrm{cm}^{3}$.
\end{abstract}

Index Terms-Epitaxial Fe/Mn/Co trilayers, ferromagnetic resonance (FMR), magnetic anisotropy.

\section{INTRODUCTION}

$\mathbf{T}$ HE study of the several aspects related to the production, characterization and applications of magnetic thin films is a very dynamic research area, employing several specific and high sensitivity experimental techniques. Among the several techniques applied to the investigation of thin-film magnetism, ferromagnetic resonance (FMR) has been used to study the magnetization, the magnetic anisotropy and the exchange coupling between the magnetic layers in a large variety of thin films and/or multilayers [1]. In particular, for monocrystalline films, the FMR measurements can be used to correlate the magnetic anisotropy to the crystalline structure of the sample. In this aspect, the FMR technique was applied, for instance, to investigate the fourfold magnetic anisotropy of epitaxial single crystalline Fe films on GaAs(110) substrate [2], sputtered Fe films on $\mathrm{MgO}$ substrate [3] and $\mathrm{Fe} / \mathrm{MgO} / \mathrm{Fe}$ trilayers grown by molecular beam epitaxy (MBE) onto $\mathrm{MgO}$ substrates [4]. In the present work, the FMR technique is used to study the magnetic anisotropy of the bcc-Fe and bcc-Co layers of monocrystalline $\mathrm{Fe} / \mathrm{Mn} / \mathrm{Co}$ trilayers MBE-grown onto cubic single crystals $\mathrm{MgO}(001)$ substrates. Previous studies [5], [6] of the structural and magnetic properties of a set of MBE-grown $\mathrm{Fe} / \mathrm{Mn} / \mathrm{Fe}$ trilayers have shown the existence of a noncollinear coupling between the Fe layers. Thus, we do also investigate if this kind of coupling can occur in cubic $\mathrm{Fe} / \mathrm{Mn} / \mathrm{Co}$ trilayers. To the best of our knowledge, studies of magnetic coupling and magnetic anisotropy of MBE-grown $\mathrm{Fe} / \mathrm{Mn} / \mathrm{Co}$ trilayers were not yet reported in the literature.

\section{SAMPLES AND EXPERIMENTS}

The magnetic anisotropy of $\mathrm{Si}(5.0 \mathrm{~nm})$ covered $\mathrm{MgO}(001) /$ $\mathrm{Cr}(2 \mathrm{~nm}) / \mathrm{Ag}(2 \mathrm{~nm}) / \operatorname{Ag}(4 \mathrm{~nm}) / \mathrm{Fe}(5.0 \mathrm{~nm}) / \mathrm{Mn}\left(\mathrm{t}_{\mathrm{Mn}}\right) /$

Manuscript received February 17, 2013; revised March 28, 2013; accepted April 19, 2013. Date of current version July 23, 2013. Corresponding author: M. S. Pessoa (e-mail: solinofis@hotmail.com).

Digital Object Identifier 10.1109/TMAG.2013.2259806
Co $(2.0 \mathrm{~nm})$ trilayers was studied by the FMR technique. Six samples with Mn layer thickness $\left(\mathrm{t}_{\mathrm{Mn}}\right)$ of $0.83,0.99,1.16$, $1.33,1.80$, and $2.20 \mathrm{~nm}$ were prepared, hereafter called samples A, B, C, D, E, and F, respectively. The monocrystalline trilayer samples were grown onto rotating $\mathrm{MgO}(001)$ substrates to ensure lateral uniformity of the film, using the KU Leuven MBE setup, with a base pressure better than $4 \times 10^{-10}$ Torr [5]. An Ag (40 nm) buffer layer was used to improve the surface roughness as well as to favor the growth of continuous Fe layers (Fe layer grown directly onto $\mathrm{MgO}$ substrate has grain-like features). The substrate temperature during $\mathrm{Fe}$ deposition was $175^{\circ} \mathrm{C}$, whereas $\mathrm{Mn}$ and $\mathrm{Co}$ were grown at $50^{\circ} \mathrm{C}$. The Si cap layer, used to minimize sample oxidation, was deposited at room temperature (RT). In situ reflection high energy electron diffraction (RHEED) analysis was performed to investigate the in-plane epitaxy of each individual layer, using electrons with energy of $10 \mathrm{keV}$ at an incident angle of about $3^{\circ}$ with respect to the film plane. It was found that all layers exhibit in-plane epitaxy, including the Co layer which seems to follow Mn-bcc-like phase. The RHEED signal intensity reduces as $t_{M n}$ increases, indicating an enhanced Mn surface roughness. XRD measurements have shown (002) texture for the $\mathrm{Fe}, \mathrm{Co}$, $\mathrm{Ag}$, and Mn layers (asymmetric Bragg peaks at $2 \theta \sim 65^{\circ}$ due to $\mathrm{Fe}$ and $\mathrm{Co}$, and sharp peaks at $2 \theta \sim 45^{\circ}$ and $55^{\circ}$ attributed, respectively, to $\mathrm{Ag}$ and $\mathrm{Mn}$; the latter with a bct-like structure [5]). Considering XRD and RHEED data, it was estimated that the in-plane lattice constant misfit between $\mathrm{Fe}, \mathrm{Mn}$, and Co is less than $2 \%$. The magnetic response of the samples was investigated using a PPMS-evercool I system. The $\mathrm{M}(\mathrm{H})$ loop recorded at RT for sample A, with the applied field along the [100] Fe easy direction, is displayed in Fig. 1. The loop indicates that the magnetizations of $\mathrm{Fe}$ and $\mathrm{Co}$ layers are noncollinearly coupled, in agreement with [5]. According to this figure, the magnetization saturation regime can be reached for field values higher than $2 \mathrm{kOe}$.

The FMR measurements were done at RT with a microwave frequency of $33.9 \mathrm{GHz}$, using a high sensitivity electron magnetic resonance spectrometer (Bruker ESP-300) with standard 


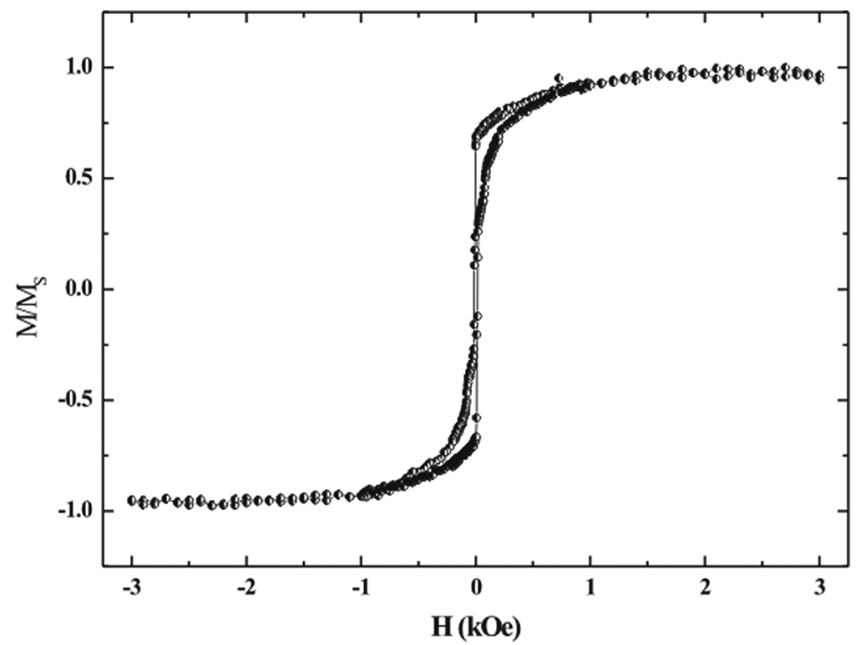

Fig. 1. $\mathrm{M}(\mathrm{H})$ loop of sample A. The direction of the applied magnetic field is the same as the [100] easy axis of the Fe layer.

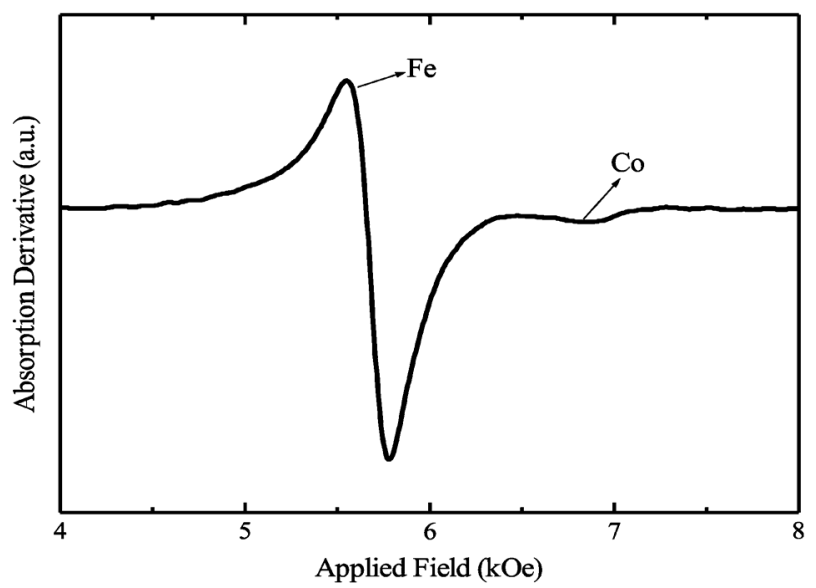

Fig. 2. In-plane FMR spectrum of sample B at microwave frequency of 33.9 $\mathrm{GHz}$. Both $\mathrm{Fe}$ and $\mathrm{Co}$ resonance modes are excited by the microwave field. Static magnetic field applied along the [110] hard axis of the Fe layer.

modulation and phase sensitive detection techniques. The swept static magnetic field was measured with a precision of $0.1 \mathrm{Oe}$ and the rotating base magnet allowed a precision of $0.1^{\circ}$ on the angle of the static magnetic field with respect to the in-plane crystallographic axis, or to the film normal. As conventional in electron magnetic resonance experiments, the microwave magnetic field was positioned perpendicular to the static magnetic field. Due to the monocrystalline structure of the films, with easy and hard in-plane magnetization axes, the characteristics of an FMR spectrum depend on the orientation of the applied static magnetic field with respect to the crystallographic axes of the Fe layer. In our experiments, the [100] and [110] axes are, respectively, the in-plane easy and hard magnetization axis of the monocrystalline Fe layer. It should also be mentioned that the [100] easy axis of magnetization of the Fe layer is parallel to the [110] in-plane axis of the $\mathrm{MgO}$ (001) substrate. The size of the FMR samples is about $2 \times 2 \mathrm{~mm}^{2}$.

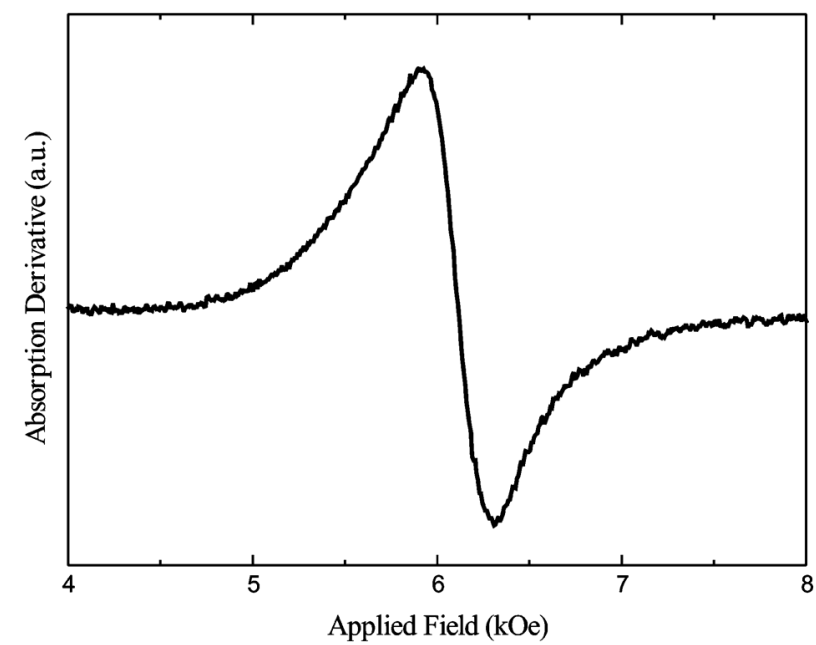

Fig. 3. In-plane FMR spectrum of sample $F$ at microwave frequency of 33.9 $\mathrm{GHz}$. A single uniform resonance mode is excited by the microwave field. Static magnetic field applied along the [110] hard axis of the Fe layer.

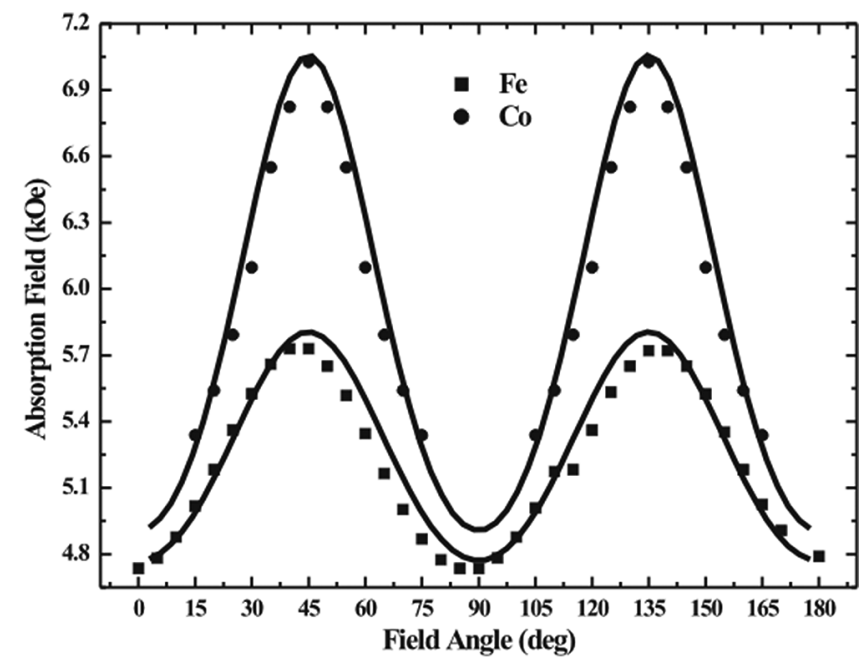

Fig. 4. Angular dependences of in-plane resonance fields of $\mathrm{Fe}$ and Co layers of sample B, at microwave frequency of $33.9 \mathrm{GHz}$ : evidence of fourfold magnetic anisotropy of both Fe and Co layers. The solid lines are fittings using the resonance and equilibrium conditions discussed in the text.

\section{FMR RESULTS}

For a frequency of $33.9 \mathrm{GHz}$, in-plane FMR spectra of samples $\mathrm{A}$ and $\mathrm{B}$ reveal that $\mathrm{Fe}$ and $\mathrm{Co}$ uniform resonance modes are independently excited by the microwave field, as illustrated in Fig. 2 for sample B. The uniform Fe and Co resonance modes are excited when the respective resonance and equilibrium conditions of the magnetization are attained. The spectra of the other four samples (with a thicker Mn layer) exhibit single uniform resonance modes, as shown for sample $\mathrm{F}$ in Fig. 3. The spectra in Figs. 2 and 3 were obtained with the static magnetic field applied along the [110] hard axis of magnetization of the Fe layer.

The angular dependence of the in-plane absorption (resonance) fields was studied for all samples. The results give evidence of a large in-plane fourfold cubic magnetic anisotropy [3], [7], as revealed by the data shown in Figs. 4 and 5. For samples A (not shown) and B, the angular dependence for $\mathrm{Fe}$ 

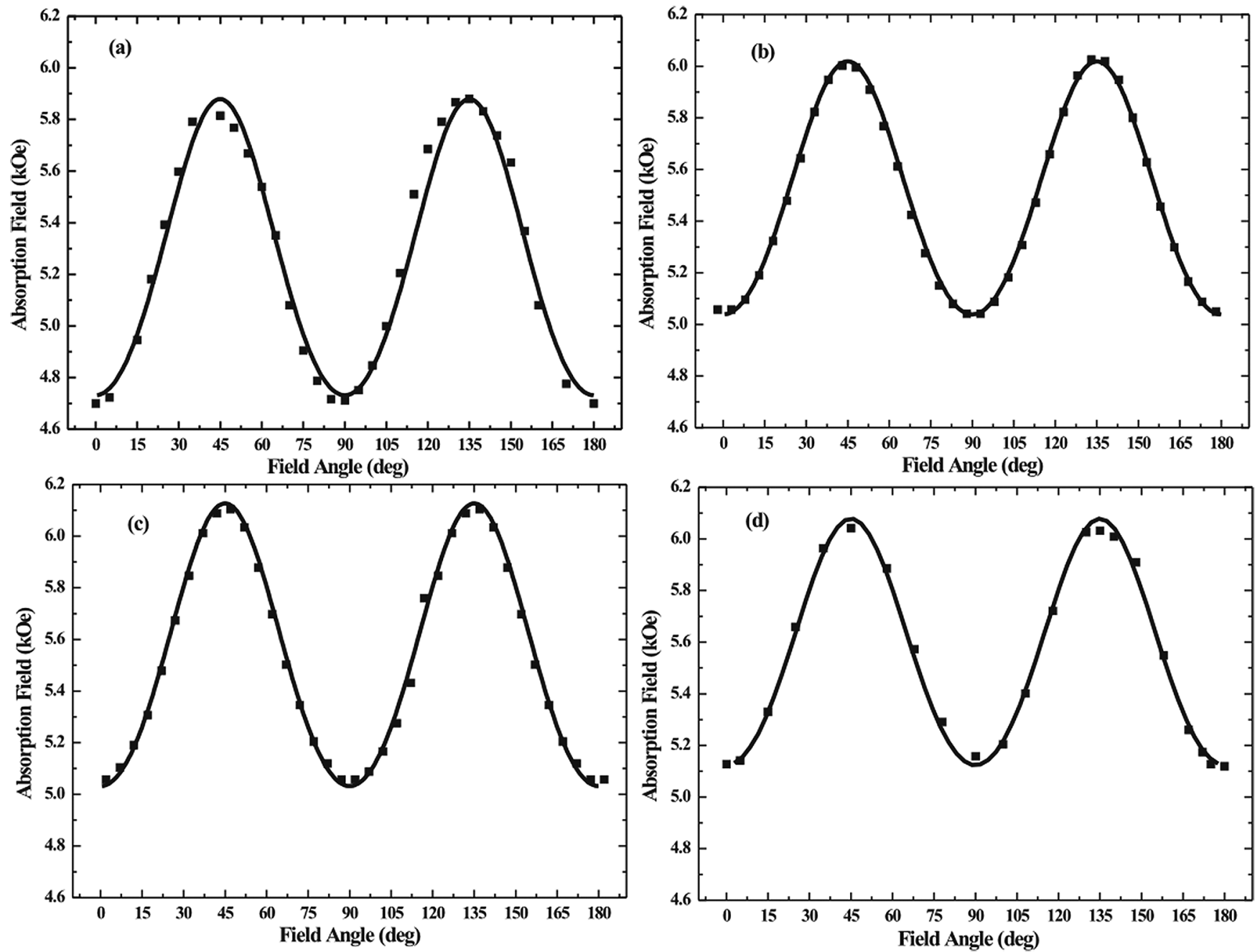

Fig. 5. Angular dependences of the in-plane resonance fields of samples (a) C; (b) D; (c) E; and (d) F, given by FMR experiments at microwave frequency of 33.9 GHz. Fourfold magnetic anisotropy revealed by the single uniform FMR mode excited by the microwave field. The solid lines are fittings using the resonance and equilibrium conditions discussed in the text.

and Co resonance modes (Fig. 4) implies the presence of a fourfold magnetic anisotropy for both Fe and Co layers. Using the FMR theory discussed below, the angular dependence of the in-plane absorption (resonance) fields with respect to the [100] axis of the Fe layer, can be fitted with the magnetic parameters deduced from the experiments.

On the other hand, it should be emphasized that FMR experiments of sample B at a microwave frequency of $9.4 \mathrm{GHz}$ (not shown) reveal that nonaligned absorption modes are also excited in $\mathrm{Fe} / \mathrm{Mn} / \mathrm{Co}$ trilayers. The excitation of nonaligned modes can be considered as evidence of noncollinear coupling between the $\mathrm{Fe}$ and $\mathrm{Co}$ ferromagnetic layers, as also found in $\mathrm{Fe} / \mathrm{Mn} / \mathrm{Fe}$ system [8]. At a microwave frequency of $9.4 \mathrm{GHz}$, the resonance fields are below $1 \mathrm{kOe}$ and in the presence of low static magnetic fields the magnetic layers are noncollinearly coupled, as suggested by the $\mathrm{M}(\mathrm{H})$ curve in Fig. 1. Additional evidence of noncollinear coupling between $\mathrm{Fe}$ and Co layers in the presence of low static magnetic fields is given by the study of the magnetization of $\mathrm{Fe} / \mathrm{Mn} / \mathrm{Co}$ trilayers and will be published elsewhere [9]. Moreover, for films with thicker Mn layers, at low microwave frequency the uniform resonance mode can only be excited with the static magnetic field applied along or maximally \pm 5 degrees away from the [110] hard axis of magnetization of the Fe layer. This behavior, also seen in $\mathrm{Fe} / \mathrm{Mn} / \mathrm{Fe}$ trilayers [8] and other systems [3], [7], [10], results from the large cubic magnetic anisotropy of the films and does not allow the study of the angular dependence of the in-plane absorption (resonance) fields at low microwave frequency. At a microwave frequency of $33.9 \mathrm{GHz}$ the static magnetic field necessary to attend the resonance condition is about $5 \mathrm{kOe}$. In the presence of high static magnetic fields the $\mathrm{Fe}$ and $\mathrm{Co}$ ferromagnetic layers are collinearly aligned and only uniform resonance modes are excited by the microwave field.

\section{FMR THEORY}

The presence of fourfold in-plane magnetic anisotropic field in the films implies that the free energy F per unit of volume can be written as

$$
\begin{aligned}
F= & -M \cdot H\left[\sin \theta \sin \theta_{H} \cos \left(\phi-\phi_{H}\right)+\cos \theta \cos \theta_{H}\right] \\
& +2 \pi M^{2} \cos ^{2} \theta+\frac{1}{4} K_{1}\left(\sin ^{2} 2 \theta+\sin ^{4} \theta \sin ^{2} 2 \phi\right) .
\end{aligned}
$$


This free energy is the result of contributions from Zeeman (first term), demagnetizing (second term), and cubic magnetic anisotropy (third term) energies. Here $\theta$ and $\theta_{H}$ are the angles of the effective magnetization $\boldsymbol{M}$ and the applied magnetic field $\boldsymbol{H}$ with respect to the normal to the film, and $\phi$ and $\phi_{H}$ are the angles with respect to the in-plane [100] axis of the Fe layer, respectively; $K_{1}$ is the fourth-order cubic anisotropy constant. The equilibrium position of $\boldsymbol{M}$ is given by the zeros of the first angular derivatives of $\boldsymbol{F}$. In our experimental situation, the in-plane equilibrium angle $\phi_{0}$ is given by the solution of

$$
H \sin \left(\phi_{0}-\phi_{H}\right)=-\frac{2 K_{1}}{M} \sin 4 \phi_{0} .
$$

The general resonance condition for the uniform FMR mode can be deduced from [10]

$$
\left(\frac{\omega}{\gamma}\right)^{2}=\frac{\gamma^{2}}{M^{2} \sin ^{2} \theta}\left[\frac{\partial^{2} F}{\partial \theta^{2}} \frac{\partial^{2} F}{\partial \phi^{2}}-\left(\frac{\partial^{2} F}{\partial \theta \partial \phi}\right)^{2}\right] .
$$

Here, $\omega$ is the resonance (angular) frequency and $\gamma=g e / 2 m$ is the gyromagnetic ratio ( $e$ is the charge and $m$ is the mass of the electron, $g$ is the spectroscopic splitting factor). According to this relation, and using the above expressions for the free energy $F$, the resonance condition for the parallel in-plane FMR measurements is

$$
\begin{array}{r}
\left(\frac{\omega}{\gamma}\right)^{2}=\left[H \cos \left(\phi_{0}-\phi_{H}\right)+4 \pi M+\frac{K_{1}}{M}\left(2-\sin ^{2} 2 \phi_{0}\right)\right] \\
\times\left[H \cos \left(\phi_{0}-\phi_{H}\right)+\frac{2 K_{1}}{M} \cos 4 \phi_{0}\right] .
\end{array}
$$

According to this theory, the resonance condition for in-plane FMR experiments directly depends on the fourfold cubic magnetic anisotropy constant $K_{1}$. The effective magnetization $M$, considered here, includes possible contributions due to interlayer coupling and interface magnetic anisotropy.

\section{ANALYSIS AND DISCUSSION}

The magnetic properties of the $\mathrm{Fe} / \mathrm{Mn} / \mathrm{Co}$ bcc-like films were deduced from the angular dependence of the in-plane absorption field of the uniform resonance mode. For samples A (not shown) and $\mathrm{B}$ (Fig. 4), the analysis was performed for both $\mathrm{Fe}$ and $\mathrm{Co}$ resonance modes. For samples C, D, E, and F (Fig. 5), the determination of the magnetic anisotropy was only done for the single uniform resonance modes observed in the FMR experiments with microwave frequency of $33.9 \mathrm{GHz}$. More specifically, the fittings of the angular dependence measurements with the appropriate equilibrium and resonance conditions discussed above yield the anisotropy constants, effective magnetizations, and g-factors listed in Table I. It should be first emphasized that the magnetic anisotropy results are in good agreement with known values for monocrystalline thin Fe [3], [7], [10], [12] and Co [13] films reported in literature. In addition, for films with thin Mn spacer, the Co magnetic anisotropy is slightly higher than that of $\mathrm{Fe}$, but both $\mathrm{Fe}$ and Co have a comparable order
TABLE I

Magnetic Parameters of Fe/Mn/Co Trilayers: Anisotropy CONSTANTS, EFFECTIVE MAGNETIZATIONS, AND G-FACTORS

\begin{tabular}{|c|c|c|c|}
\hline \multicolumn{5}{|c|}{ Sample A - Fe/Mn/Co } \\
\hline & $\mathrm{K}_{1}\left(\mathrm{erg} / \mathrm{cm}^{3}\right)$ & $4 \pi \mathrm{M}(\mathrm{kG})$ & g-factor \\
\hline Fe & $2.0 \pm 0.1 \times 10^{5}$ & $14.3 \pm 0.2$ & $2.06 \pm 0.01$ \\
\hline Co & $2.2 \pm 0.1 \times 10^{5}$ & $12.6 \pm 0.2$ & $2.20 \pm 0.01$ \\
\hline \multicolumn{5}{|c|}{ Sample B $-\mathbf{F e} / \mathrm{Mn} / \mathbf{C o}$} \\
\hline & $\mathrm{K}_{1}\left(\mathrm{erg} / \mathrm{cm}^{3}\right)$ & $4 \pi \mathrm{M}(\mathrm{kG})$ & g-factor \\
\hline Fe & $4.5 \pm 0.1 \times 10^{5}$ & $19.0 \pm 0.2$ & $2.09 \pm 0.01$ \\
\hline Co & $6.4 \pm 0.1 \times 10^{5}$ & $12.3 \pm 0.2$ & $2.20 \pm 0.01$ \\
\hline \multicolumn{5}{|c|}{ Fe-Co } \\
\hline Sample & $\mathrm{K}_{1}\left(\mathrm{erg} / \mathrm{cm}^{3}\right)$ & $4 \pi \mathrm{M}(\mathrm{kG})$ & g-factor \\
\hline C & $4.9 \pm 0.1 \times 10^{5}$ & $18.6 \pm 0.2$ & $2.10 \pm 0.01$ \\
\hline D & $4.0 \pm 0.1 \times 10^{5}$ & $17.6 \pm 0.2$ & $2.10 \pm 0.01$ \\
\hline E & $4.4 \pm 0.1 \times 10^{5}$ & $17.4 \pm 0.2$ & $2.10 \pm 0.01$ \\
\hline F & $3.8 \pm 0.1 \times 10^{5}$ & $17.3 \pm 0.2$ & $2.10 \pm 0.01$ \\
\hline
\end{tabular}

of magnitude due to their bcc-like features, as suggested by RHEED and XRD data.

The FMR experiments at a frequency of $33.9 \mathrm{GHz}$ reveal that for films with thicker Mn layers, only a single uniform resonance mode is excited by the microwave field. To a first approximation, this can be attributed to: (i) large fractions of $\mathrm{Fe}$ and $\mathrm{Co}$ atoms affected by $\mathrm{Mn}$ atoms at $\mathrm{Fe} / \mathrm{Mn}$ and $\mathrm{Mn} / \mathrm{Co}$ interfaces, respectively, and (ii) the magnetization saturation regime reached by $\mathrm{Fe}$ and $\mathrm{Co}$ layers in the presence of high static magnetic fields. It should be mentioned that, as suggested by RHEED briefly discussed above, the roughness of the Mn layer increases with increasing $\mathrm{t}_{\mathrm{Mn}}$. This roughness effect may favor a Co atomic inter-diffusion at the $\mathrm{Mn} / \mathrm{Co}$ interface (alloying process). Moreover, all samples were prepared with $1 \mathrm{~nm}$ of ${ }^{57} \mathrm{Fe}$ layer deposited at the $\mathrm{Fe} / \mathrm{Mn}$ interface and ${ }^{57} \mathrm{Fe}$-Mössbauer spectroscopy suggests that a relative great fraction of $\mathrm{Fe}$ atoms ( $40 \%$ of the total spectrum area) display magnetic properties that deviate from metallic $\mathrm{Fe}$. This effect is due to nonequivalent configurations for $\mathrm{Fe}$ sites at the $\mathrm{Fe} / \mathrm{Mn}$ interface, i.e., $\mathrm{Fe}$ atoms at the corner, step-edges, flat regions. This is also a possibility of a disordered $\mathrm{Fe} / \mathrm{Mn}$ alloy formation at the interface; this problem will be discussed elsewhere [9]. Thus, mainly in $\mathrm{Mn} / \mathrm{Co}$ interface, one may assume that for large Mn thickness the interface effects reduce the difference between the effective magnetizations of $\mathrm{Fe}$ and Co layers. In view of this, at a microwave frequency of $33.9 \mathrm{GHz}$ the in-plane FMR experiments cannot reveal independent resonance modes of $\mathrm{Fe}$ and Co coupled layers. The wide resonance lines must result from the overlap of $\mathrm{Fe}$ and Co resonance lines.

Finally, due to the rectangular shape of the samples used for the FMR experiments, a correction term dependent on the dimensions of the samples and demagnetizing effects [14] could be added to the resonance condition (3). We argue here, however, that to deduce from the FMR experiments a true contribution from a shape parameter, it would be necessary to compare the results given by rectangular and circular samples (exhibiting homogeneous demagnetizing effects). This could be an extension of this work. Otherwise, the addition of the shape parameter 
could be compensated by a different g-factor to deduce the same values of anisotropy constants.

\section{CONCLUSION}

The MBE-grown $\mathrm{Fe} / \mathrm{Mn} / \mathrm{Co}$ trilayers present the in-plane fourfold cubic magnetic anisotropy field characteristic of monocrystalline Fe films. Bcc-Fe and bcc-Co uniform FMR modes are excited in samples with a thin Mn layer (0.83 and $0.99 \mathrm{~nm}$ ), implying that the Co layer also exhibits a fourfold cubic magnetic anisotropy. Magnetic crystalline anisotropies of the bcc-Fe and bcc-Co phases were deduced as $4.5 \times 10^{5}$ and $6.4 \times 10^{5} \mathrm{erg} / \mathrm{cm}^{3}$, respectively. These values can be used, for example, to fit $\mathrm{M}(\mathrm{H})$ curves, where usual bulk-like values are assumed in the fitting procedure. Samples with a thick Mn layer $(>1.16 \mathrm{~nm})$ do not exhibit independent $\mathrm{Fe}$ and Co uniform FMR modes; this behavior can be explained by assuming an atomic interdiffusion at the Fe/Mn and $\mathrm{Mn} / \mathrm{Co}$ interfaces (enhanced in Mn/Co interface as the Mn thickness increases). In general, noncollinear coupling of $\mathrm{Fe}$ and $\mathrm{Co}$ layers occurs in films with thin Mn spacer, in the presence of low static magnetic fields. The noncollinear coupling was not observed for thicker Mn layer, as reveal the FMR data obtained at a microwave frequency of $33.9 \mathrm{GHz}$.

\section{ACKNOWLEDGMENT}

This work was supported by the Brazilian agencies $\mathrm{CNPq}$, CAPES and FAPES, the Belgium Fund for Scientific Research, Flanders (FWO) and the KU Leuven Research Fund (GOA/ 2009/006).

\section{REFERENCES}

[1] J. Lindner and K. Baberschke, "Ferromagnetic resonance in coupled ultrathin films," J. Phys. Cond. Mater., vol. 15, pp. S465-S478, Jan. 2003.
[2] G. A. Prinz, G. T. Rado, and J. J. Krebs, "Magnetic properties of singlecrystal $\{110\}$ iron films grown on GaAs by molecular beam epitaxy," J. Appl. Phys., vol. 53, pp. 2087-2091, Mar. 1982.

[3] Y. V. Goryunov, N. N. Garif'yanov, G. G. Khaliulin, I. A. Garifullin, L. R. Taginov, F. Schreiber, T. Mühge, and H. Zabel, "Magnetic anisotropies of sputtered Fe films on $\mathrm{MgO}$ substrates," Phys. Rev. B, vol. 52 , pp. $13450-13458$, Nov. 1995.

[4] E. Popova, C. Tiusan, A. Schuhl, F. Gendron, and N. A. Lesnik, "Ferromagnetic resonance in the epitaxial system $\mathrm{Fe} / \mathrm{MgO} / \mathrm{Fe}$ with coupled magnetic layers," Phys. Rev. B, vol. 74, pp. 224415-1-224415-7, Dec. 2006.

[5] E. C. Passamani, B. Croonenborghs, B. Degroote, and A. Vantomme, "Interface and bulk properties of Fe/Mn sandwich structures," Phys. Rev. B, vol. 67, pp. 174424-1-174424-8, May 2003.

[6] B. R. Segatto, "Origem do acoplamento não-colinear nas superestruturas tipo $\mathrm{Fe} / \mathrm{Mn} / \mathrm{Fe}$ crescidas por molecular beam epitaxy," (in Portuguese) M.Sc. thesis, Departamento de Física, Univ. Fed. do Espírito Santo, Vitoria, 2007.

[7] T. Tolinski, K. Lenz, J. Lindner, E. Kosubek, K. Baberschke, D. Spoddig, and R. Meckenstock, "Magnetic anisotropies and dispersion relation of epitaxial Fe/InAs(001) films," Solid State Commun., vol. 128, pp. 385-389, Aug. 2003.

[8] M. S. Pessoa, "Anisotropia magnética em tricamadas epitaxiais $\mathrm{Fe} / \mathrm{Mn} / \mathrm{Fe}$," (in Portuguese) M.Sc. dissertation, Inst. de Física, Univ. Fed. Goiás, Goiânia, 2010.

[9] T. C. de Freitas, E. C. Passamani, S. Couet, K. Temst, and A. Vantomme, to be published.

[10] E. C. da Silva, R. Meckenstock, O. Von Geisau, R. Kordecki, J. Pelzl, J. A. Wolf, and P. Grünberg, "Ferromagnetic resonance investigations of anisotropy fields of Fe(001) epitaxial layers," J. Magn. Magn. Mater., vol. 121 , pp. 528-531, 1993.

[11] J. Smit and H. G. Beljers, "Ferromagnetic resonance absorption in $\mathrm{BaFe}_{12} \mathrm{O}_{19}$ a highly anisotropic crystal," Phil. Res. Rep., vol. 10, pp. 113-130, Feb. 1955.

[12] Y. Gondo, S. Usami, K. Itoh, H. Konno, and Z. Funatogawa, "Ferromagnetic resonance in single-crystal thin films of iron," J. Appl. Phys., vol. 34, pp. 1081-1082, Apr. 1963.

[13] M. J. M. Pires, A. A. C. Cotta, M. D. Martins, A. M. A. Silva, and W. A. A. Macedo, "Fourfold magnetic anisotropy in a Co film on $\mathrm{MgO}(001)$," J. Magn. Magn. Mater., vol. 323, pp. 789-793, Nov. 2010.

[14] D. S. Schmool and J. M. Barandiarán, "New phenomena in the study of shape effects in ferromagnetic resonance," J. Magn. Magn. Mater., vol. 191, pp. 211-224, Aug. 1998. 